Supporting Information for

\title{
Understanding the Origin of the Breakdown of the Stokes- Einstein Relation in Supercooled Water at Different Temperature-Pressure Conditions
}

Vikas Dubey ${ }^{\dagger}$ Shakkira Erimban ${ }^{\dagger}$ Sandipa Indra, Snehasis Daschakraborty*

Department of Chemistry, Indian Institute of Technology Patna, Bihar 801106, India.

\author{
AUTHOR INFORMATION \\ Corresponding Author \\ *snehasis@iitp.ac.in
}


Table 1. Production trajectory duration $t_{t r a j}$ for simulations at different $T$-P state points.

\begin{tabular}{ccccccc}
\hline T (K) & $\mathbf{0 ~ M P a}$ & $\mathbf{5 0} \mathbf{M P a}$ & $\mathbf{1 0 0} \mathbf{M P a}$ & $\mathbf{1 5 0} \mathbf{M P a}$ & $\mathbf{2 0 0} \mathbf{M P a}$ & $\mathbf{2 5 0} \mathbf{M P a}$ \\
\hline $\mathbf{2 2 0}$ & 200 & 200 & 200 & 200 & 200 & 200 \\
$\mathbf{2 3 0}$ & 200 & 200 & 200 & 200 & 200 & 200 \\
$\mathbf{2 4 0}$ & 200 & 200 & 200 & 200 & 200 & 200 \\
$\mathbf{2 5 0}$ & 100 & 100 & 100 & 100 & 100 & 100 \\
$\mathbf{2 6 0}$ & 100 & 100 & 100 & 100 & 100 & 100 \\
$\mathbf{2 7 0}$ & 100 & 50 & 50 & 50 & 50 & 50 \\
$\mathbf{2 8 0}$ & 50 & 50 & 50 & 50 & 50 & 50 \\
$\mathbf{3 0 0}$ & 50 & 50 & 50 & 50 & 50 & 50 \\
$\mathbf{3 2 0}$ & 50 & 50 & 50 & 50 & 50 & 50 \\
$\mathbf{3 4 0}$ & 50 & 50 & 50 & 50 & 50 & 50 \\
\hline
\end{tabular}



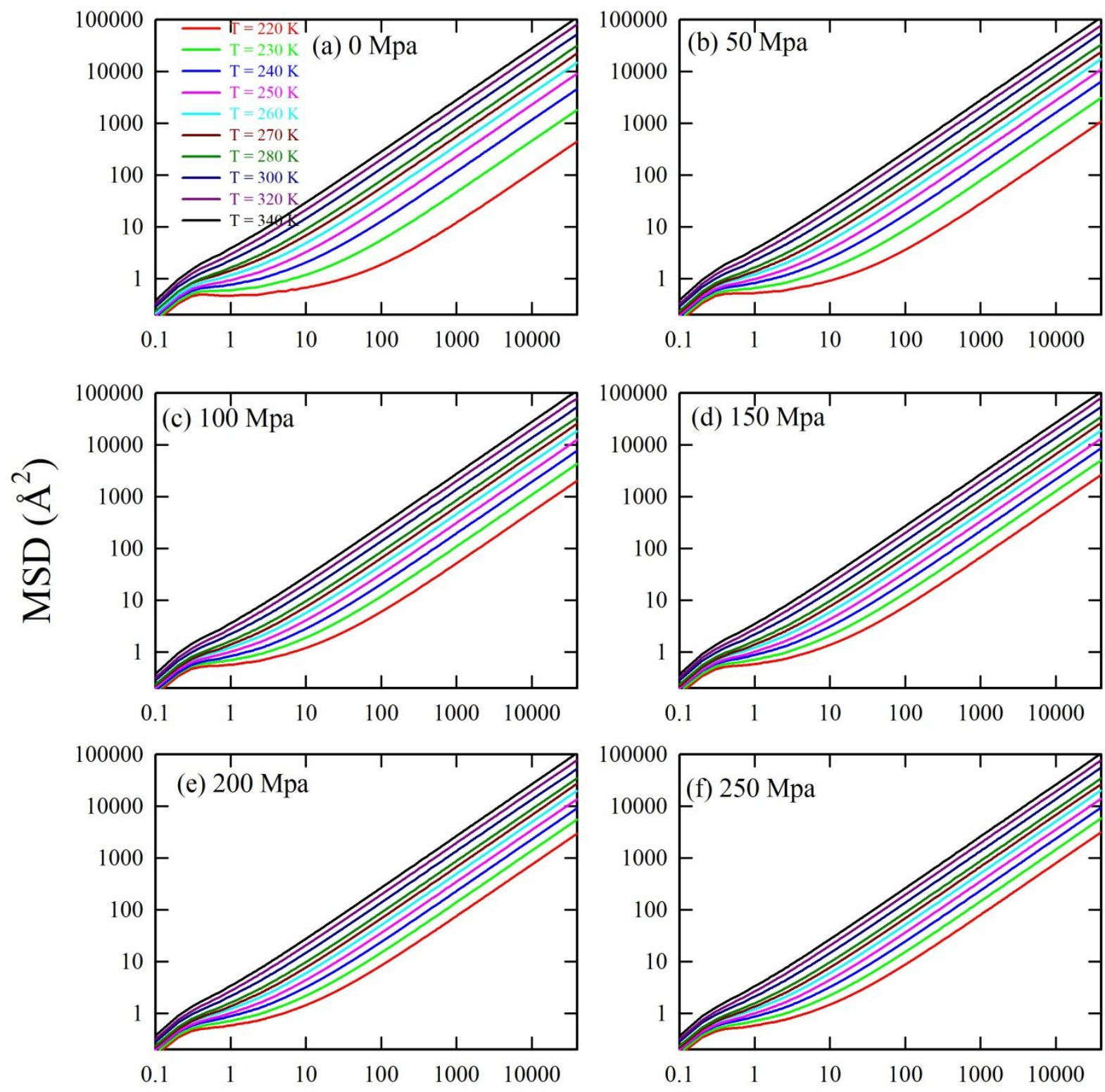

\section{time (ps)}

Figure S1. Mean square displacement (MSD) as functions of time at different temperatures and pressures. 

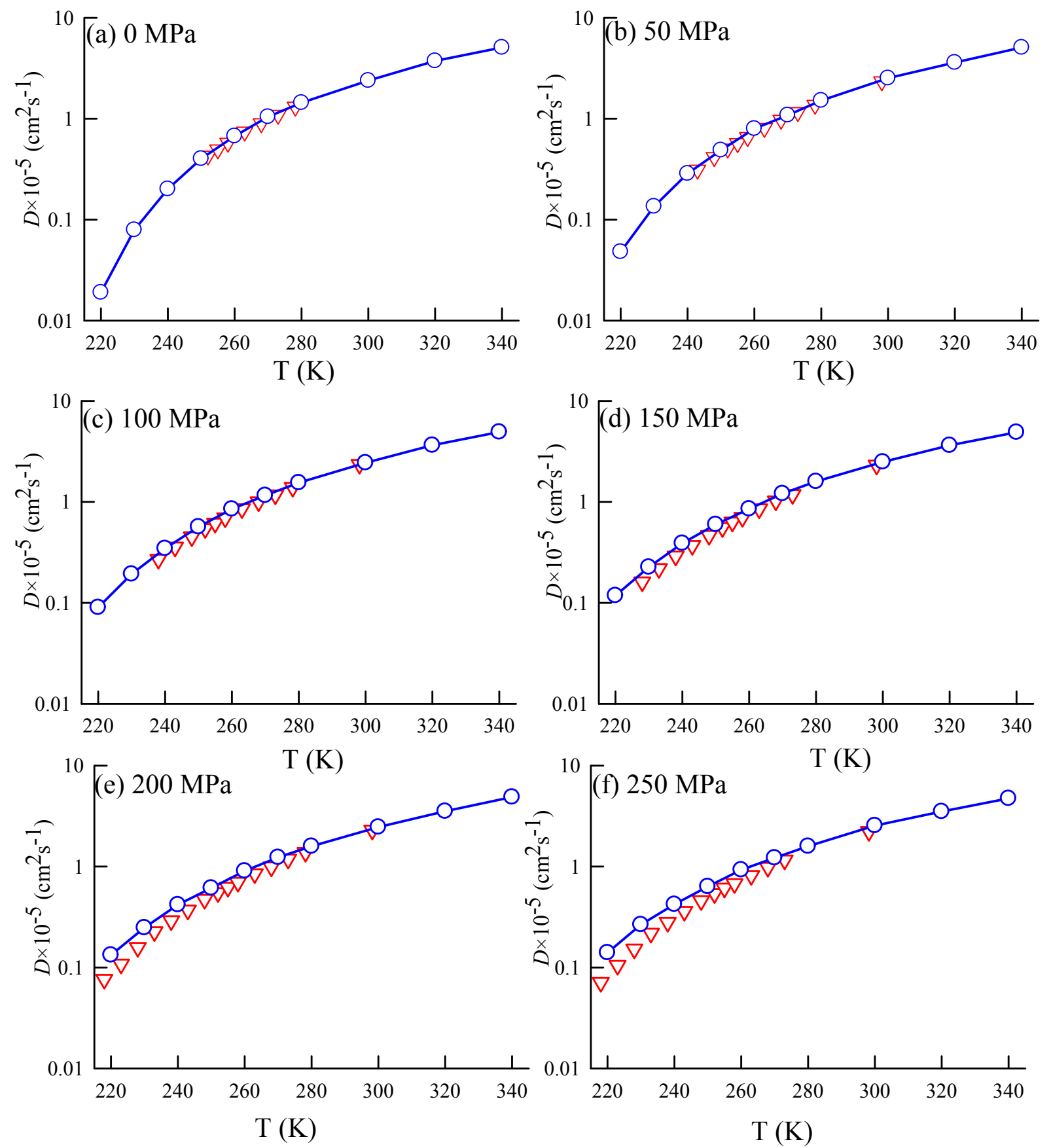

Figure S2: Comparison between the current simulated and experimental ${ }^{1,2}$ self diffusion coefficients. 


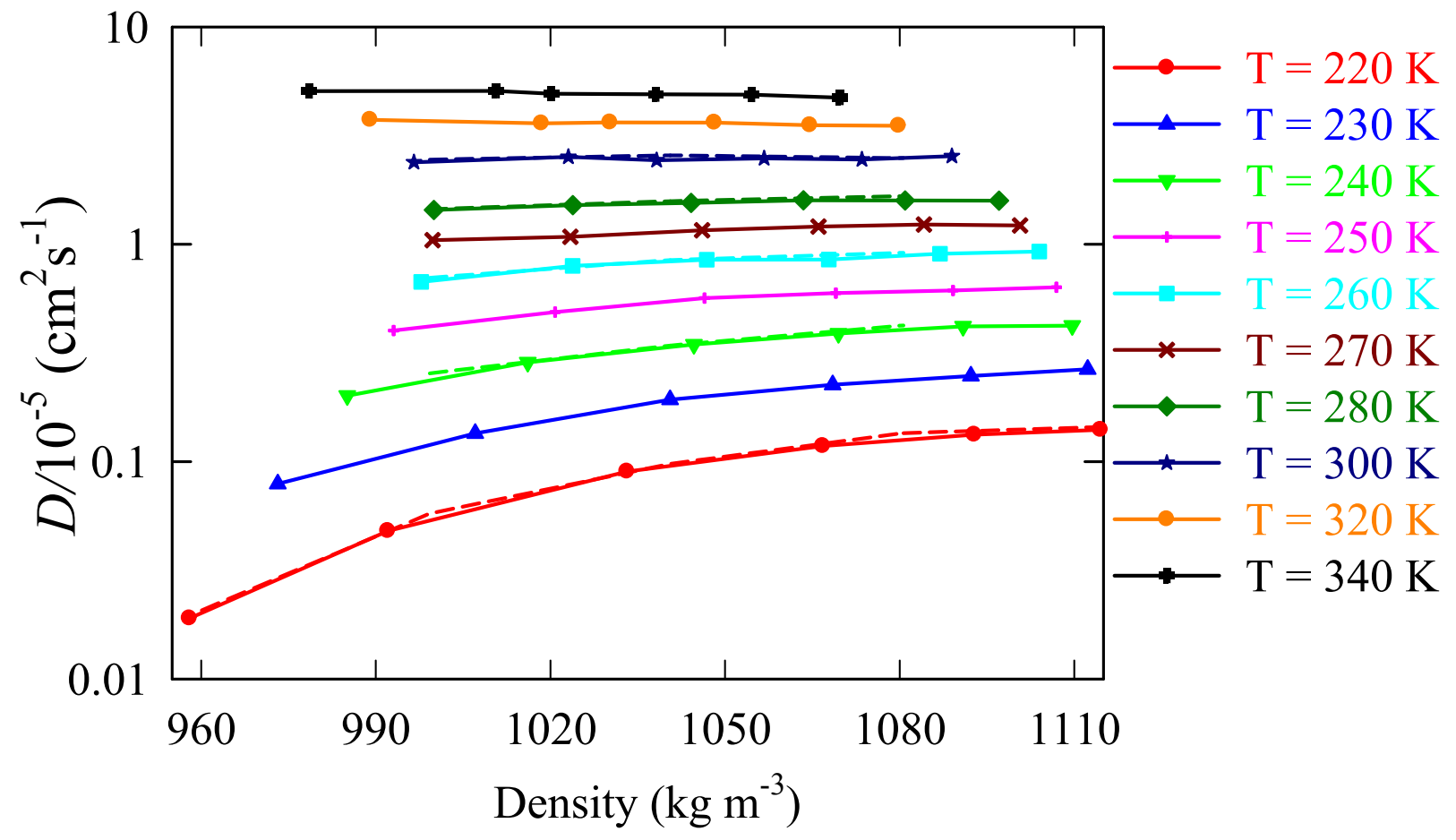

Figure S3:. Comparison between the present simulated diffusion coefficient values and the simulated values by Montero de Hijes et al. ${ }^{3}$ versus density at different temperatures. 

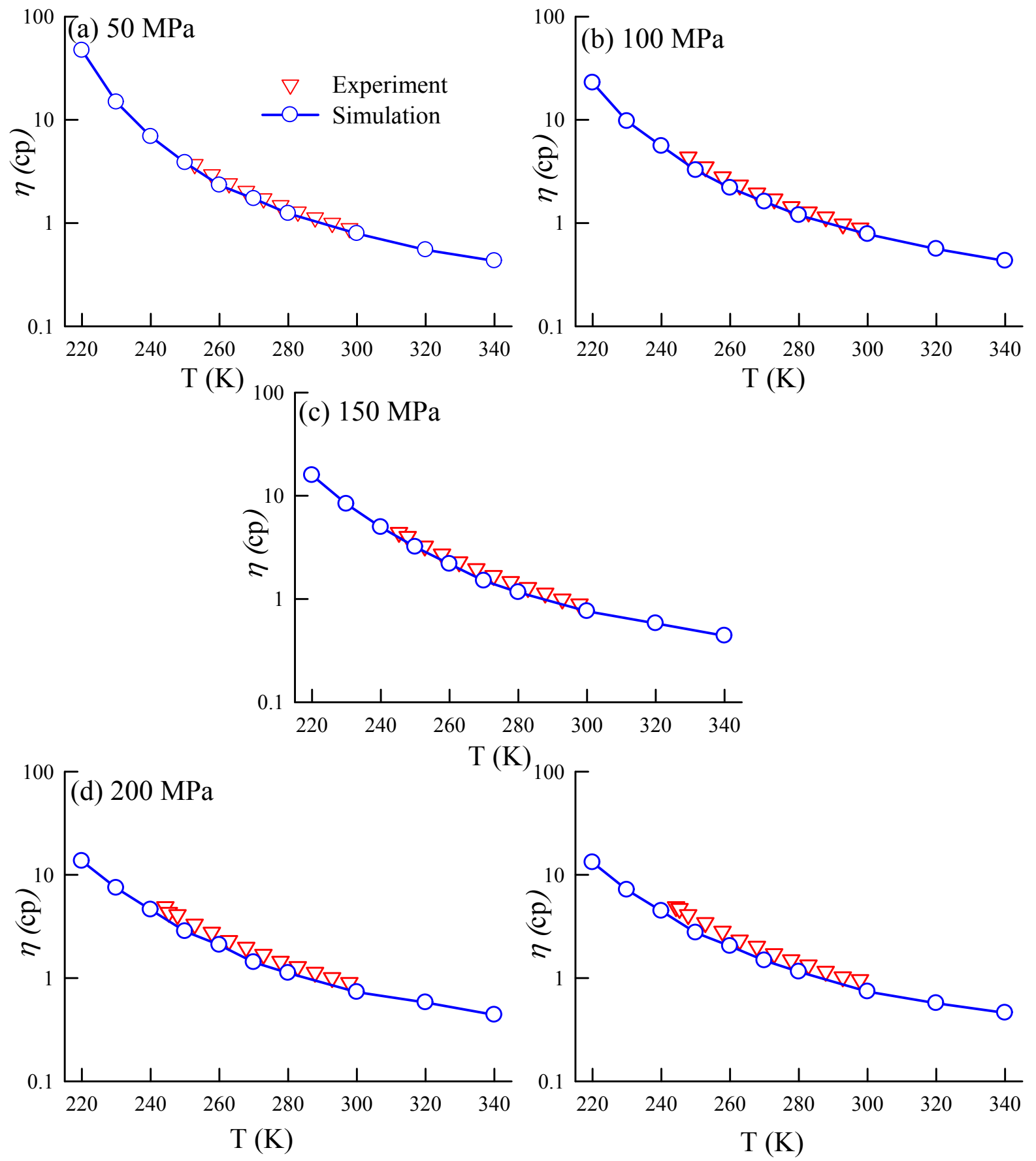

Figure S4: Comparison between the present simulated and experimental viscosity coefficients ${ }^{4}$ at different $T-P$ state points. 


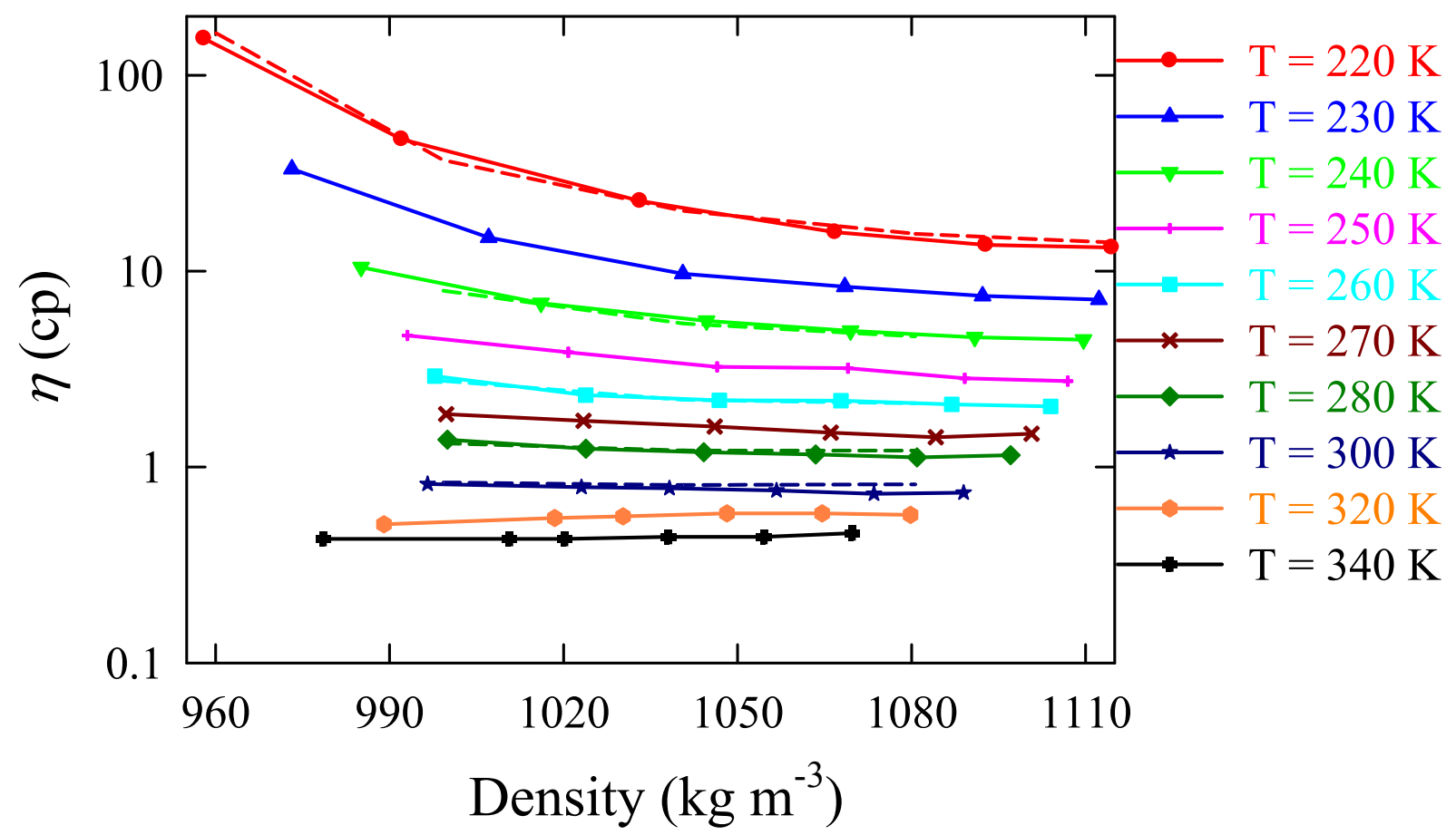

Figure S5. Comparison between the present simulated viscosity coefficient values and the simulated values by Montero de Hijes et al. ${ }^{3}$ versus density at different temperatures. 
Table S2. Temperature and pressure-dependent values of different simulated parameters.

\begin{tabular}{|c|c|c|c|c|c|c|c|c|c|}
\hline $\mathrm{T}(\mathrm{K})$ & $\begin{array}{c}\mathrm{P} \\
(\mathrm{MPa})\end{array}$ & $\begin{array}{c}\left(D / 10^{-5}\right) \\
\left(\mathrm{cm}^{2} / \mathrm{sec}\right)\end{array}$ & $\eta(\mathrm{cp})$ & $\begin{array}{c}t^{*} \\
(\mathrm{ps})\end{array}$ & $\begin{array}{c}r_{2} \\
(\mathrm{~nm})\end{array}$ & $\begin{array}{l}v_{\text {Jump }} \\
\left(\mathrm{ns}^{-1}\right)\end{array}$ & $\begin{array}{c}\lambda_{\text {Jump }}^{2} \\
\left(\AA^{2}\right)\end{array}$ & $\begin{array}{c}D_{\text {Jump }} / 10^{-5} \\
\left(\mathrm{~cm}^{2} / \mathrm{sec}\right)\end{array}$ & $\begin{array}{l}D_{\text {Res }} / 10^{-5} \\
\left(\mathrm{~cm}^{2} / \mathrm{sec}\right)\end{array}$ \\
\hline \multirow{6}{*}{220} & 0 & 0.019 & 154 & 90.0 & 0.233 & 0.47 & 8.30 & 0.0069 & 0.0121 \\
\hline & 50 & 0.048 & 47.1 & 33.3 & 0.225 & 0.99 & 7.12 & 0.0125 & 0.0355 \\
\hline & 100 & 0.090 & 22.8 & 16.7 & 0.22 & 1.42 & 6.50 & 0.0165 & 0.0735 \\
\hline & 150 & 0.118 & 15.8 & 12.2 & 0.21 & 1.82 & 5.90 & 0.0192 & 0.0988 \\
\hline & 200 & 0.133 & 13.6 & 10.3 & 0.21 & 1.75 & 5.74 & 0.0181 & 0.1149 \\
\hline & 250 & 0.140 & 13.2 & 9.70 & 0.20 & 2.17 & 5.33 & 0.0208 & 0.1192 \\
\hline \multirow{6}{*}{230} & 0 & 0.079 & 31.2 & 21.2 & 0.230 & 1.52 & 7.39 & 0.0197 & 0.0593 \\
\hline & 50 & 0.135 & 14.8 & 12.1 & 0.23 & 1.83 & 7.05 & 0.0230 & 0.1120 \\
\hline & 100 & 0.193 & 9.69 & 8.50 & 0.225 & 2.16 & 6.59 & 0.0256 & 0.1674 \\
\hline & 150 & 0.226 & 8.33 & 7.10 & 0.22 & 2.42 & 6.25 & 0.0271 & 0.1989 \\
\hline & 200 & 0.248 & 7.47 & 5.56 & 0.21 & 2.65 & 5.63 & 0.0270 & 0.2210 \\
\hline & 250 & 0.266 & 7.16 & 5.40 & 0.21 & 2.54 & 5.61 & 0.0251 & 0.2409 \\
\hline \multirow{6}{*}{240} & 0 & 0.201 & 10.5 & 8.40 & 0.24 & 2.25 & 7.61 & 0.0306 & 0.1704 \\
\hline & 50 & 0.286 & 6.87 & 6.00 & 0.24 & 2.08 & 7.39 & 0.0276 & 0.2584 \\
\hline & 100 & 0.346 & 5.57 & 5.30 & 0.24 & 2.09 & 7.31 & 0.0276 & 0.3184 \\
\hline & 150 & 0.389 & 4.96 & 3.90 & 0.22 & 3.12 & 6.13 & 0.0344 & 0.3546 \\
\hline & 200 & 0.419 & 4.60 & 3.50 & 0.22 & 2.64 & 6.05 & 0.0287 & 0.3903 \\
\hline & 250 & 0.422 & 4.47 & 3.40 & 0.22 & 2.77 & 6.02 & 0.0259 & 0.3961 \\
\hline \multirow{6}{*}{250} & 0 & 0.401 & 4.70 & 5.1 & 0.25 & 2.77 & 8.05 & 0.0403 & 0.3607 \\
\hline & 50 & 0.488 & 3.85 & 3.5 & 0.215 & 1.75 & 8.02 & 0.0254 & 0.4626 \\
\hline & 100 & 0.566 & 3.25 & 3.2 & 0.225 & 4.56 & 6.42 & 0.0531 & 0.5129 \\
\hline & 150 & 0.597 & 3.19 & 2.9 & 0.23 & 3.28 & 6.60 & 0.0392 & 0.5578 \\
\hline & 200 & 0.613 & 2.84 & 2.8 & 0.23 & 3.00 & 6.57 & 0.0360 & 0.5770 \\
\hline & 250 & 0.634 & 2.75 & 2.6 & 0.21 & 4.72 & 5.54 & 0.0568 & 0.5772 \\
\hline \multirow{6}{*}{260} & 0 & 0.672 & 2.91 & 3.0 & 0.26 & 2.52 & 8.48 & 0.0387 & 0.6333 \\
\hline & 50 & 0.796 & 2.33 & 2.6 & 0.255 & 2.61 & 8.08 & 0.0384 & 0.7576 \\
\hline & 100 & 0.850 & 2.19 & 2.6 & 0.25 & 3.43 & 7.47 & 0.0465 & 0.8035 \\
\hline & 150 & 0.851 & 2.18 & 2.3 & 0.25 & 2.31 & 7.66 & 0.0322 & 0.8188 \\
\hline & 200 & 0.905 & 2.09 & 2.2 & 0.24 & 2.93 & 7.07 & 0.0377 & 0.8673 \\
\hline & 250 & 0.927 & 2.04 & 2.2 & 0.24 & 2.18 & 7.57 & 0.0244 & 0.9026 \\
\hline \multirow{6}{*}{270} & 0 & 1.045 & 1.86 & 2.3 & 0.28 & 1.86 & 9.65 & 0.0324 & 1.0126 \\
\hline & 50 & 1.082 & 1.72 & 2.1 & 0.26 & 3.15 & 8.36 & 0.0480 & 1.0340 \\
\hline & 100 & 1.160 & 1.61 & 1.9 & 0.25 & 3.51 & 7.70 & 0.0494 & 1.1106 \\
\hline & 150 & 1.207 & 1.50 & 1.9 & 0.25 & 3.26 & 7.66 & 0.0456 & 1.1614 \\
\hline & 200 & 1.234 & 1.42 & 1.6 & 0.24 & 3.13 & 7.02 & 0.0402 & 1.1938 \\
\hline & 250 & 1.220 & 1.48 & 1.6 & 0.24 & 2.64 & 6.98 & 0.0358 & 1.1842 \\
\hline
\end{tabular}




\begin{tabular}{|c|c|c|c|c|c|c|c|c|c|}
\hline \multirow{6}{*}{280} & 0 & 1.439 & 1.38 & 1.6 & 0.27 & 2.63 & 8.93 & 0.0427 & 1.3963 \\
& 50 & 1.513 & 1.24 & 1.5 & 0.27 & 2.08 & 8.38 & 0.0335 & 1.4795 \\
& 100 & 1.550 & 1.19 & 1.4 & 0.25 & 3.46 & 7.62 & 0.0483 & 1.5017 \\
& 150 & 1.594 & 1.16 & 1.3 & 0.26 & 2.13 & 7.81 & 0.0303 & 1.5637 \\
& 200 & 1.590 & 1.12 & 1.2 & 0.22 & 2.93 & 6.94 & 0.0374 & 1.5526 \\
& 250 & 1.587 & 1.15 & 1.2 & 0.22 & 6.69 & 5.92 & 0.0677 & 1.5193 \\
\hline \multirow{6}{*}{300} & 0 & 2.384 & 0.82 & 1.1 & 0.3 & 1.05 & 10.63 & 0.0203 & 2.3637 \\
& 50 & 2.523 & 0.79 & 1.1 & 0.3 & 0.86 & 10.58 & 0.0166 & 2.5064 \\
& 100 & 2.438 & 0.78 & 1.0 & 0.28 & 1.27 & 9.23 & 0.0215 & 2.4165 \\
& 150 & 2.485 & 0.76 & 1.0 & 0.28 & 1.03 & 9.19 & 0.0173 & 2.4677 \\
& 200 & 2.456 & 0.73 & 1.0 & 0.28 & 0.84 & 8.56 & 0.0210 & 2.4350 \\
& 250 & 2.544 & 0.74 & 1.0 & 0.27 & 0.97 & 8.53 & 0.0177 & 2.5263 \\
& 0 & 3.735 & 0.51 & 1.1 & 0.35 & 0.47 & 14.23 & 0.0125 & 3.7225 \\
& 50 & 3.598 & 0.54 & 1.0 & 0.33 & 0.52 & 12.62 & 0.0122 & 3.5858 \\
& 100 & 3.637 & 0.56 & 1.0 & 0.33 & 0.45 & 12.43 & 0.0104 & 3.6266 \\
& 150 & 3.628 & 0.58 & 1.0 & 0.32 & 0.37 & 12.36 & 0.0084 & 3.6196 \\
& 200 & 3.529 & 0.58 & 1.0 & 0.32 & 0.52 & 11.77 & 0.0090 & 3.5200 \\
& 250 & 3.507 & 0.57 & 0.9 & 0.30 & 0.46 & 10.34 & 0.0096 & 3.4974 \\
\hline \multirow{6}{*}{340} & 0 & 5.069 & 0.43 & 1.0 & 0.41 & 0.09 & 18.76 & 0.0034 & 5.0656 \\
& 50 & 5.072 & 0.43 & 0.9 & 0.39 & 0.08 & 16.86 & 0.0025 & 5.0695 \\
& 100 & 4.925 & 0.43 & 0.9 & 0.38 & 0.08 & 16.30 & 0.0023 & 4.9227 \\
& 150 & 4.897 & 0.44 & 0.9 & 0.37 & 0.09 & 15.54 & 0.0025 & 4.8945 \\
& 200 & 4.880 & 0.44 & 0.9 & 0.36 & 0.11 & 14.61 & 0.0030 & 4.8770 \\
& 250 & 4.732 & 0.46 & 0.9 & 0.36 & 0.10 & 14.59 & 0.0024 & 4.7296 \\
\hline \multirow{6}{*}{30} & & & & & & & & &
\end{tabular}




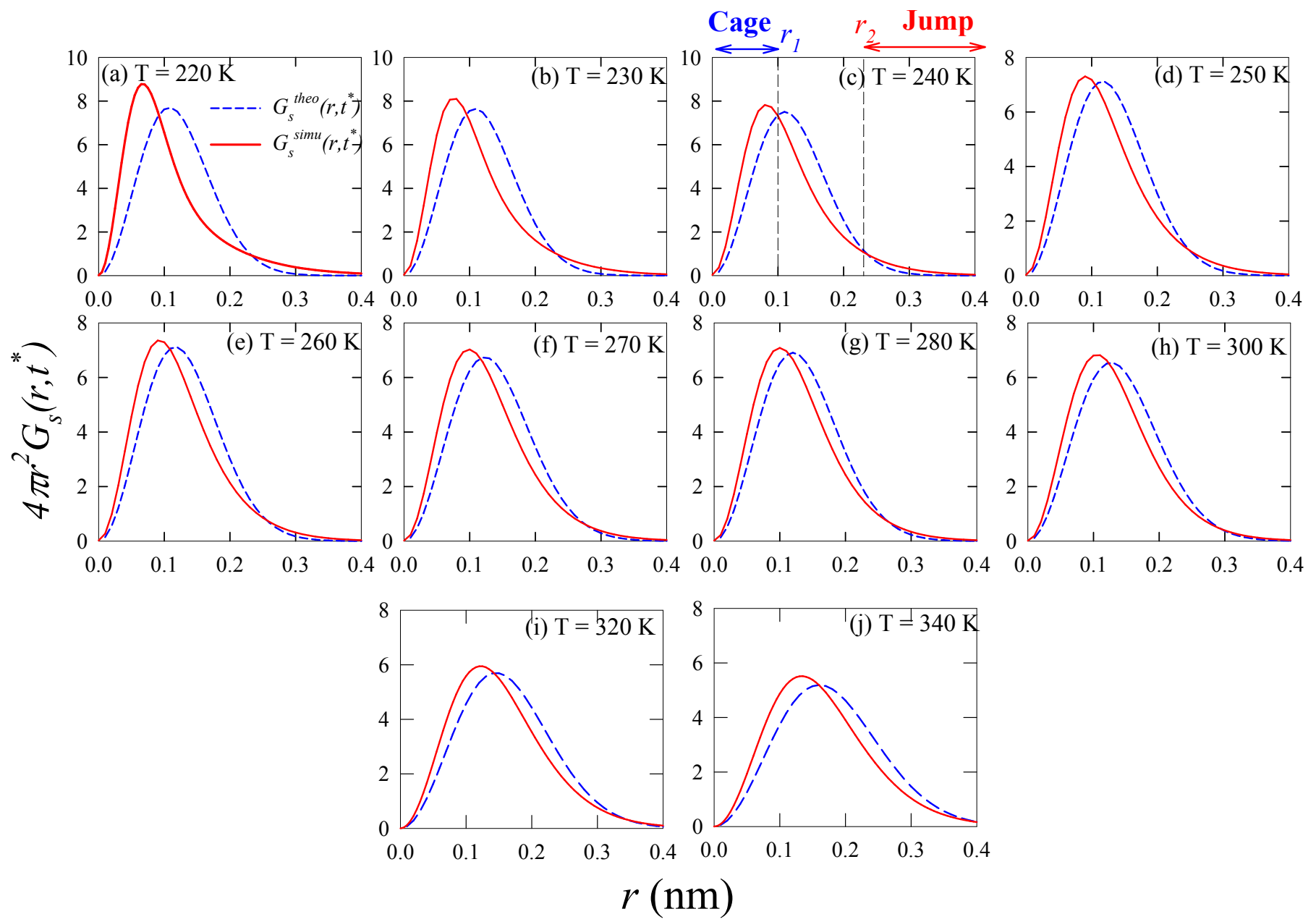

Figure S6. Comparison between the self-part of the van Hove correlation function $G_{s}\left(r, t^{*}\right)$ (solid line) and the corresponding theoretical Gaussian distribution $\left(G_{S}^{\text {theo }}\left(r, t^{*}\right)\right)$ (dashed line) at time $t^{*}$ for all the temperatures and $0 \mathrm{MPa}$ pressure. 
(b) $50 \mathrm{MPa}$

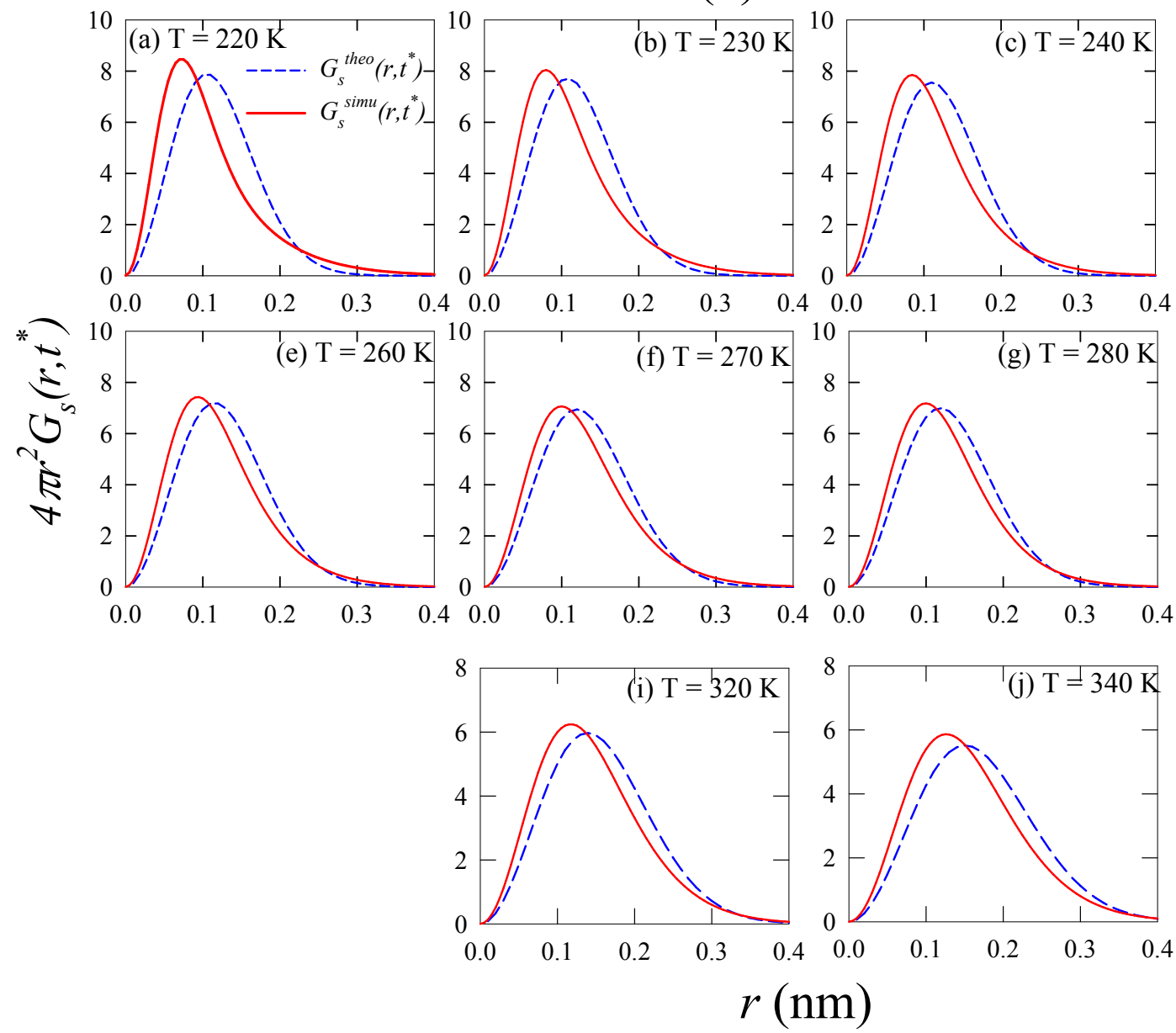

Figure S6 (continued). Comparison between the self-part of the van Hove correlation function $G_{s}$ $\left(r, t^{*}\right)$ (solid line) and the corresponding theoretical Gaussian distribution $\left(G_{S}^{t h e o}\left(r, t^{*}\right)\right)$ (dashed line) at time $t^{*}$ for all the temperatures and $50 \mathrm{MPa}$ pressure. 
(c) $100 \mathrm{MPa}$
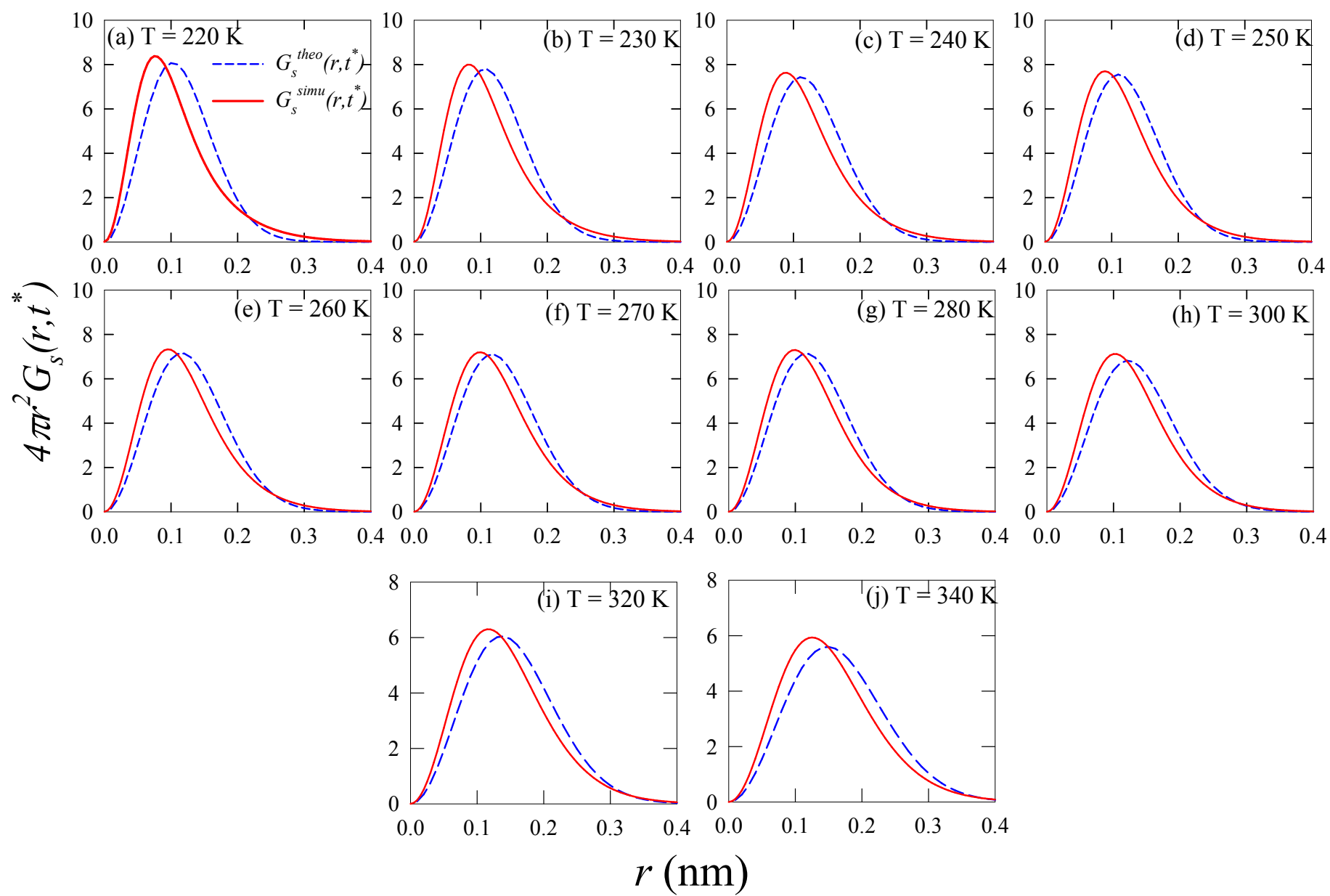

Figure S6 (continued). Comparison between the self-part of the van Hove correlation function $G_{s}$ $\left(r, t^{*}\right)$ (solid line) and the corresponding theoretical Gaussian distribution $\left(G_{S}^{\text {theo }}\left(r, t^{*}\right)\right)$ (dashed line) at time $t^{*}$ for all the temperatures and $100 \mathrm{MPa}$ pressure. 


\section{(d) $150 \mathrm{MPa}$}

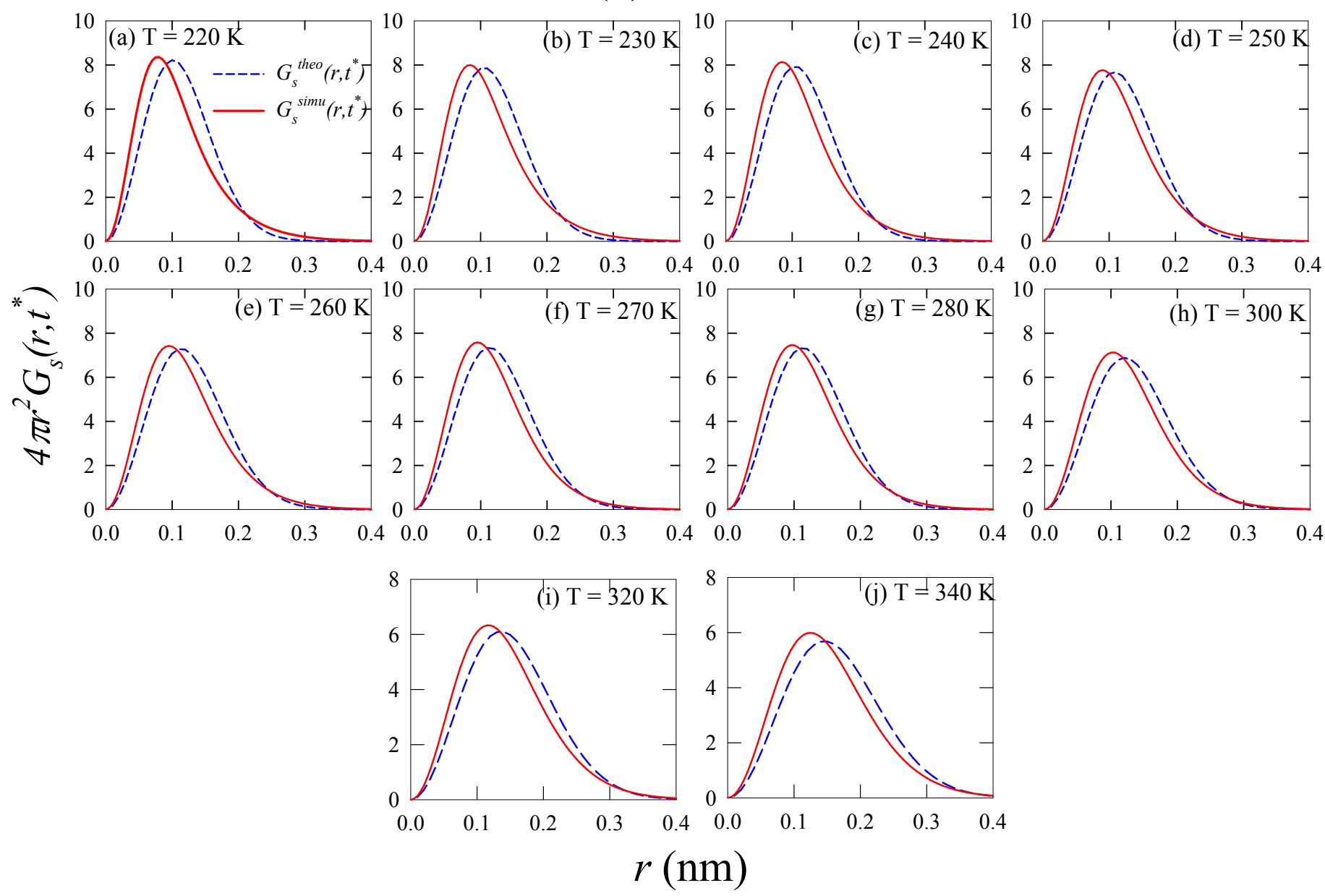

Figure S6 (continued). Comparison between the self-part of the van Hove correlation function $G_{s}$ $\left(r, t^{*}\right)$ (solid line) and the corresponding theoretical Gaussian distribution $\left(G_{S}^{t h e o}\left(r, t^{*}\right)\right)$ (dashed line) at time $t^{*}$ for all the temperatures and $150 \mathrm{MPa}$ pressure. 
(e) $200 \mathrm{MPa}$

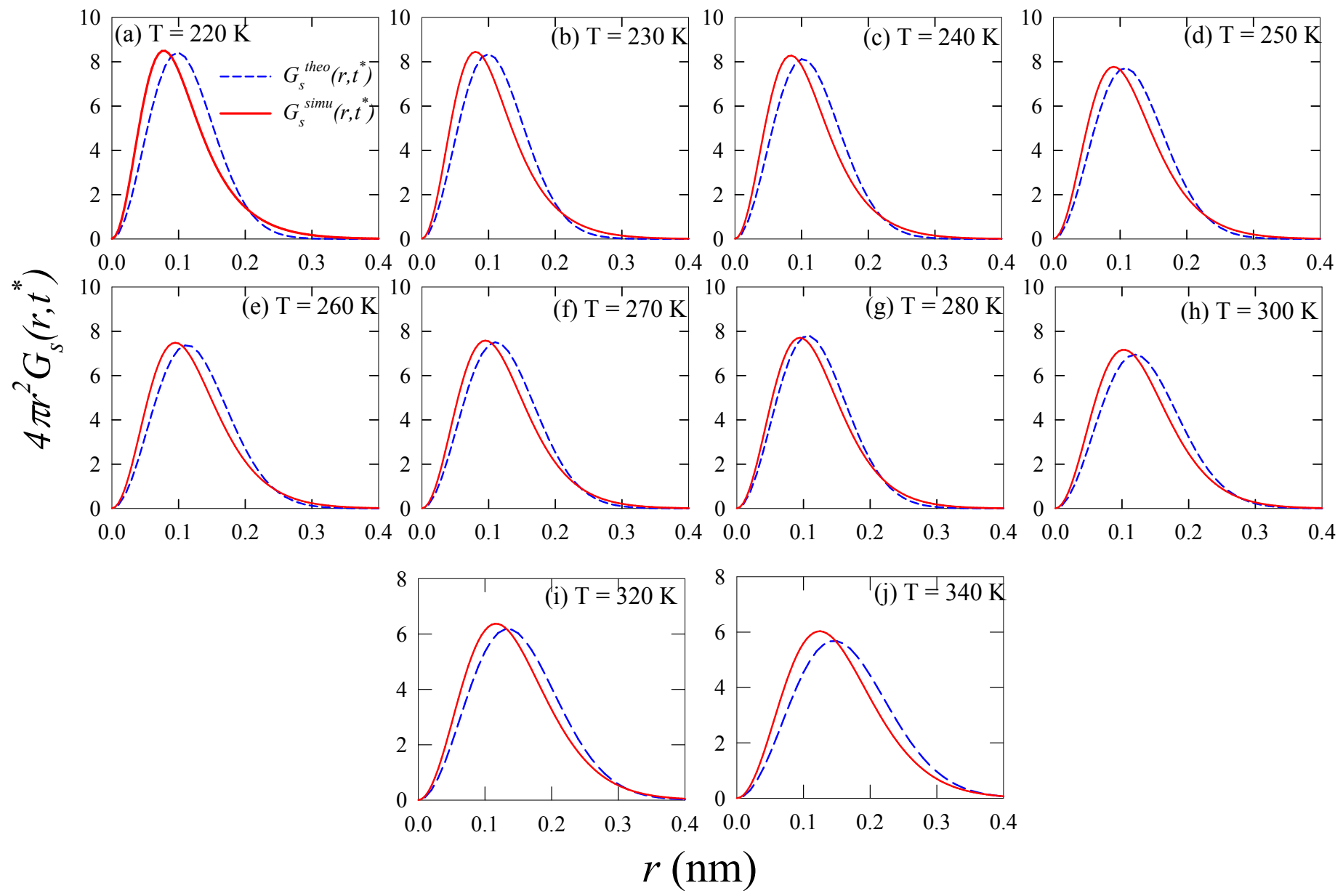

Figure S6 (continued). Comparison between the self-part of the van Hove correlation function $G_{S}$ $\left(r, t^{*}\right)$ (solid line) and the corresponding theoretical Gaussian distribution $\left(G_{S}^{t h e o}\left(r, t^{*}\right)\right)$ (dashed line) at time $t^{*}$ for all the temperatures and $200 \mathrm{MPa}$ pressure. 
(f) $250 \mathrm{MPa}$

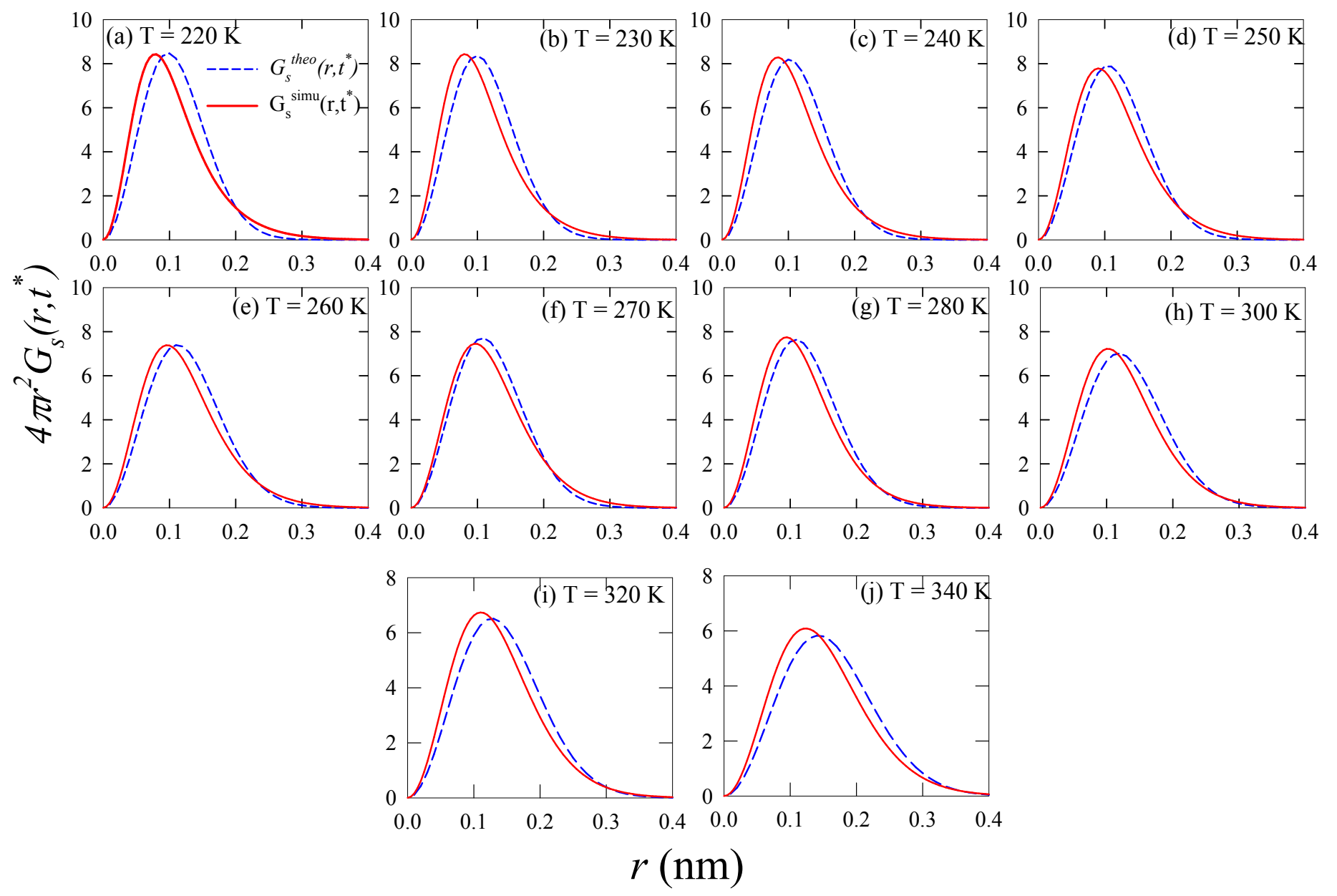

Figure S6 (continued). Comparison between the self-part of the van Hove correlation function $G_{s}$ $\left(r, t^{*}\right)$ (solid line) and the corresponding theoretical Gaussian distribution $\left(G_{S}^{\text {theo }}\left(r, t^{*}\right)\right)$ (dashed line) at time $t^{*}$ for all the temperatures and $250 \mathrm{MPa}$ pressure. 

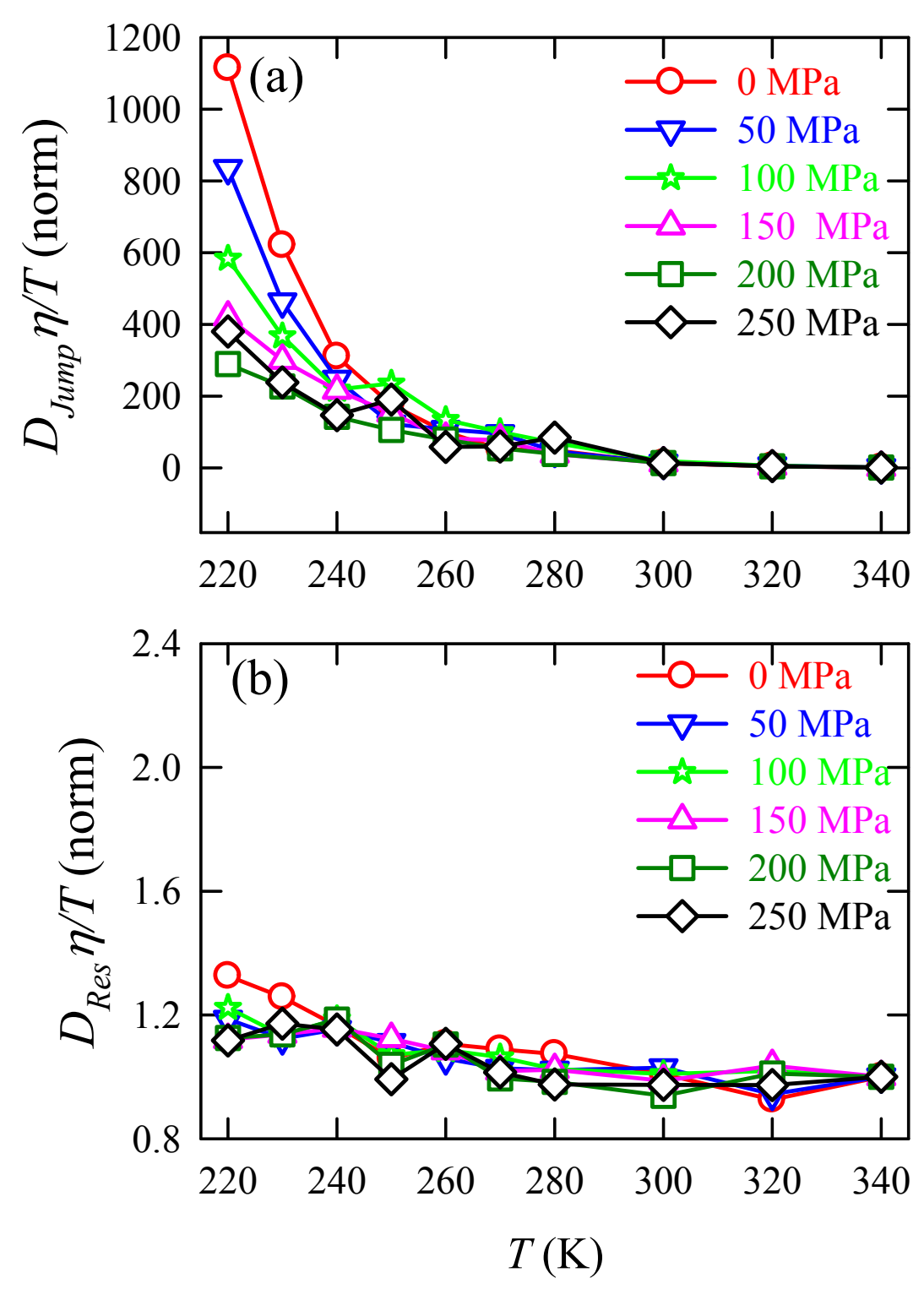

Figure S7. (a)Normalized $D_{\text {Jump }} \eta / T$ (normalized by the value at $\mathrm{T}=340 \mathrm{~K}$ ) as a function of temperature for different pressures. (b) Normalized $D_{R e s} \eta / T$ (normalized by the value at $\mathrm{T}=340$ $\mathrm{K})$ as a function of temperature and the effect of pressures on the plot. 

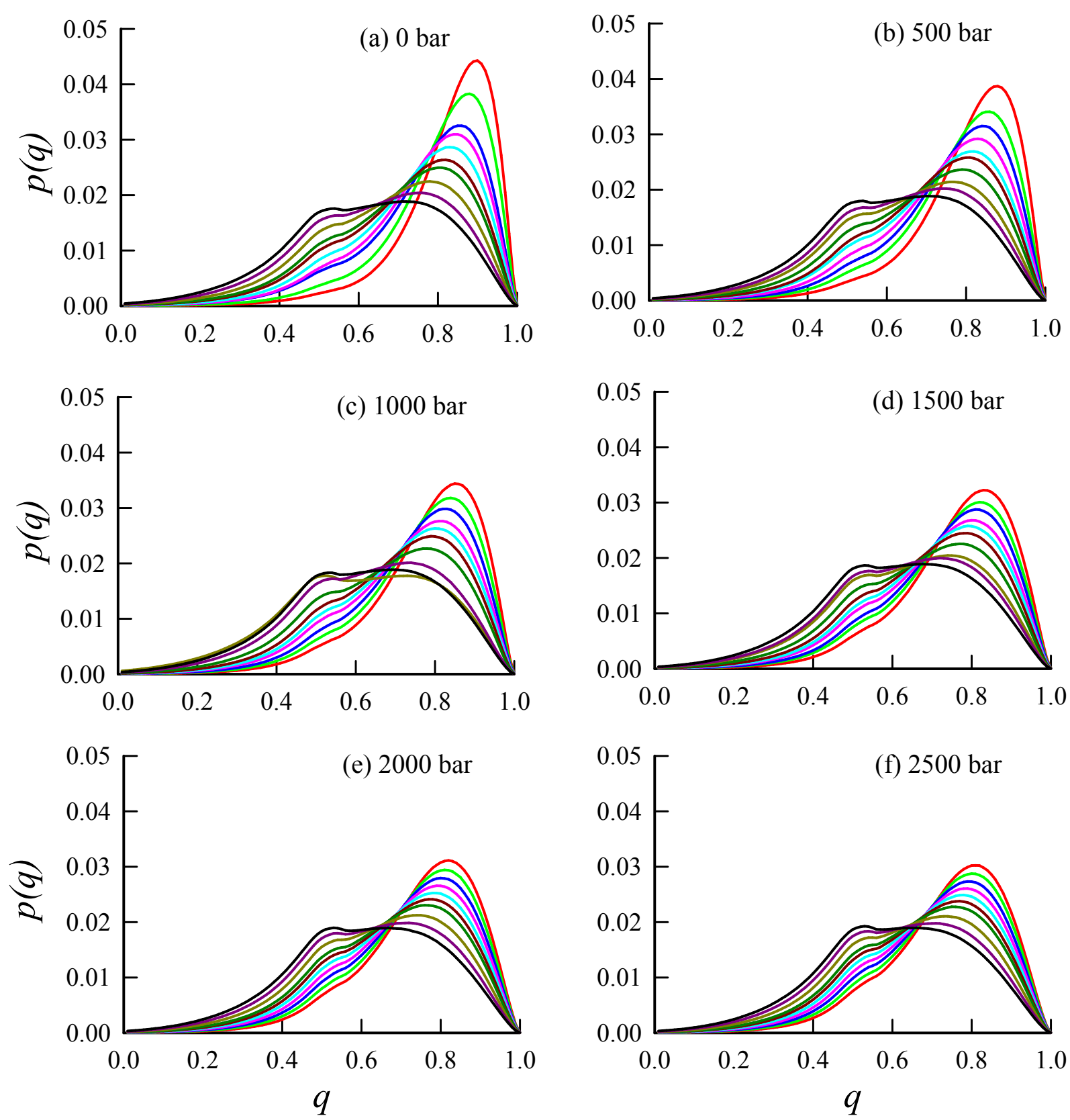

Figure S8. Distribution of tetrahedral order parameter $q$ for different $T-P$ state points. 


\section{REFERENCES}

1. Harris, K. R.; Newitt, P. J. Self-Diffusion of Water at Low Temperatures and High Pressure. J. Chem. Eng. Data 1997, 42 (2), 346-348.

2. Tazi, S.; Botan, A.; Salanne, M.; Marry, V.; Turq, P.; Rotenberg, B. Diffusion Coefficient and Shear Viscosity of Rigid Water Models. Journal of physics. Condensed matter : an Institute of Physics journal 2012, 24, 284117.

3. Montero de Hijes, P.; Sanz, E.; Joly, L.; Valeriani, C.; Caupin, F. Viscosity and SelfDiffusion of Supercooled and Stretched Water from Molecular Dynamics Simulations. J. Chem. Phys. 2018, 149 (9), 094503.

4. Singh, L. P.; Issenmann, B.; Caupin, F. Pressure Dependence of Viscosity in Supercooled Water and a Unified Approach for Thermodynamic and Dynamic Anomalies of Water. Proc Natl Acad Sci USA 2017, 114 (17), 4312. 